\title{
Erratum to: Effect of a mixed reality-based intervention on arm, hand, and finger function on chronic stroke
}

\author{
Carolina Colomer ${ }^{1,2}$, Roberto Llorens ${ }^{1,3^{*}}$, Enrique Noé ${ }^{1}$ and Mariano Alcañiz $z^{3,4}$
}

\section{Erratum}

The original article [1] mistakenly omitted a key affiliation for the author, Carolina Colomer. The authors would therefore like to state the affiliation of 'Universidad Autónoma de Barcelona, Barcelona, Spain' as the second affiliation for Dr Colomer.

Furthermore, the authors would also like to add a statement to the Acknowledgements sub-section stating:

"This work has been developed within the framework of a medical doctorate at the Universidad Autónoma de Barcelona."

\begin{abstract}
Author details
${ }^{1}$ Servicio de Neurorehabilitación y Daño Cerebral de los Hospitales NISA. Fundación Hospitales NISA, Valencia, Spain. ²Universidad de Autónoma Barcelona, Barcelona, Spain. ${ }^{3}$ Instituto Interuniversitario de Investigación en Bioingeniería y Tecnología Orientada al Ser Humano, Universitat Politècnica de València, Camino de Vera s/n, Valencia 46022, Spain. ${ }^{4}$ Ciber, Fisiopatología Obesidad y Nutrición, CB06/03 Instituto de Salud Carlos III, Av. Sos Baynat s/n, University of Jaume I, Castellón 12071, Spain.
\end{abstract}

Received: 8 February 2017 Accepted: 8 February 2017

Published online: 14 February 2017

\section{References}

1. Colomer C, et al. Effect of a mixed reality-based intervention on arm, hand, and finger function on chronic stroke. J Neuroeng Rehabil. 2016;13:45.

\footnotetext{
* Correspondence: rllorens@labhuman.com

'Servicio de Neurorehabilitación y Daño Cerebral de los Hospitales NISA. Fundación Hospitales NISA, Valencia, Spain

${ }^{3}$ Instituto Interuniversitario de Investigación en Bioingeniería y Tecnología Orientada al Ser Humano, Universitat Politècnica de València, Camino de Vera s/n, Valencia 46022, Spain
} 